\title{
Interspecific Hybridization of the Korean Native Bumblebee Bombus hypocrita sapporoensia and the European Bumblebee B. terrestris
}

\author{
Hyung Joo Yoon ${ }^{1, *}$, In Gyun Park ${ }^{1}$, Kyeong Yong Lee ${ }^{1,2}$, Mi Ae Kim ${ }^{1}$, and Byung Rae Jinn ${ }^{2, * *}$ \\ ${ }^{1}$ Department of Agricultural Biology, National Academy of Agricultural Science, Suwon 441-100, Republic of Korea \\ ${ }^{2}$ College of Natural Resources and Life Science, Dong-A University, Busan 604-714, Republic of Korea
}

(Received 27 August 2011; Accepted 11 September 2011)

The large bumblebee Bombus terrestris, indigenous to Europe and adjacent areas and used extensively for high-value crop pollination, has been artificially introduced to several parts of the world. Here we show the occurrence of interspecific hybridization between the bumblebee species $B$. hypocrita sapporoensis and $B$. terrestris under laboratory conditions. The mating and oviposition percentages resulting from the interspecific hybridization of a $B$. terrestris queen with a $B$. $h$. sapporoensis male were higher than those resulting from the intraspecific mating of $B$. $h$. sapporoensis. Furthermore, a competitive copulation experiment indicated that the mating of $B$. $h$. sapporoensis males with $B$. terrestris queens was $\mathbf{1 . 2}$-fold more frequent than the mating of these males with $B$. $h$. sapporoensis queens. The interspecific hybridization of a $B$. terrestris queen with a $B$. $h$. sapporoensis male produced either $B$. terrestris workers or the $B$. terrestris male phenotype, and the hybridization of a $B$. $h$. sapporoensis queen with a $B$. terrestris male produced $B$. $h$. sapporoensis males. Our results indicated that interspecific hybridization occurred between $B$. h. sapporoensis and $B$. terrestris. These results suggest that such hybridization will have a negative competitive impact and will cause genetic contamination of native bumblebees.

Key words: Bombus hypocrita sapporoensis, B. terrestris, Bumblebee, Interspecific hybridization, Pollinator

To whom the correspondence addressed

* Department of Agricultural Biology, National Academy of Agricultural Science, Suwon 441-100, Korea.

E-mail: yoonhj1023@korea.kr

**College of Natural Resources and Life Science, Dong-A University, Busan 604-714, Korea.

E-mail: brjin@dau.ac.kr

\section{Introduction}

Pollination is an ecosystem service because wild pollinators, in particular wild bees, contribute significantly to the pollination of a large array of crops (Morandin and Winston, 2005; Greenleaf and Kremen, 2006; Winfree et al., 2007). Commercially managed bees are also available for pollination services and are used in large commercial fields, small gardens, or enclosures such as greenhouses and screen houses (Free, 1993; Dag and Kammer, 2001). Bumblebees are important pollinators for wild flora and agricultural production. The introduction of bumblebees into greenhouses for pollination has become widespread in recent years, and demand increases annually. Bumblebees give farmers the opportunity to decrease their pollination labor costs and offer a good crop yield, both in quantity and in quality (Velthuis and van Doorn, 2006). Bumblebees are more effective pollinators than honeybees in cloudy weather and in small areas, such as greenhouses. Bumblebees also tend to devote themselves primarily to the crops within the greenhouse, whereas honeybees are apt to escape en masse to the outside. Bumblebees are particularly effective at pollinating Solanaceae, including the tomato and eggplant.

The large bumblebee Bombus terrestris is naturally distributed in Europe and adjacent territories, including England, most of Scotland, the north coast of Africa, southern Scandinavia, major Mediterranean islands, and some Atlantic islands (Madeira and the Canary Islands) (Estoup et al., 1996; Chittka et al., 2004; Velthuis and van Doorn, 2006). Since 1987, B. terrestris has been available commercially in portable boxes for crop pollination (Mitsuhata, 2000). Colonies of $B$. terrestris have been imported into many countries, including Korea, Japan, China, Taiwan, Mexico, Chile, Argentina, Uruguay, South Africa, Morocco, and Tunisia (Dafni, 1998). There has been some anxiety associated with the introduction of $B$. 
terrestris into greenhouses because it is highly invasive (Hingston et al., 2002), could possibly escape from greenhouses, and could have negative effects through competition (Ono and Wada, 1996; Velthuis and van Doorn, 2006; Inoue et al., 2008; Inoue and Yokoyama, 2010), genetic contamination by hybridization (Rhymer and Simberloff, 1996; Ono, 1997; Kanbe et al., 2008) with native bumblebees, or through naturalization (Donovan and Wier 1978; Dafni and Shimida, 1996; Ruz and Herrera 2001, Hingston et al., 2002). The competitive displacement of native pollinators and the invasion of native vegetation by $B$. terrestris have already been recorded in Tasmania, Australia (Semmens et al., 1993; Hingston and McQuillan, 1998, 1999; Hingston et al., 2002). In Israel, there has been a decline in the numbers of honeybees and solitary bees associated with the range expansion of $B$. terrestris (Dafni and Shimida, 1996). B. terrestris has also colonized Japan, where it escaped from greenhouses in Monbetsu in the Hidaka region of Hokkaido in 1996 after its introduction in 1991 (Iwasaki, 1995; Goka, 1998; Washitani, 1998; Inoue and Yokoyama, 2010). For this reason, the governments of Canada and the USA prohibit the introduction of foreign bumblebee species, and at present, a native bumblebee, B. impatiens, is used for commercial pollination in North America (Velthuis and van Doorn, 2006). B. terrestris was designated an invasive alien species under the "Invasive Alien Species Act" in 2006 in Japan. According to this law, farmers wishing to use $B$. terrestris for pollination must be issued a permit by the responsible minister (Yoneda et al., 2008). In Korea, $B$. terrestris was first introduced in 1994 . From early May 2002 through 2010, overwintering queens of B. terrestris were caught in several regions (Yoon et al., 2009, 2010). We are studying the artificial year-round mass rearing of B. ignitus and B. hypocrita sapporoensis, Korean native bumblebees, because those species are the most reliable native bumblebees for crop pollination (Yoon et al., 1999, 2002, 2004, 2010).

In the present study, we investigated the possible competition or genetic contamination of the native bumblebee, $B$. h. sapporoensis, resulting from hybridization with the imported B. terrestris. Our study focused on hybridization between these bumblebee species under laboratory conditions.

\section{Materials and Methods}

\section{Experimental animals}

The experimental animals were 2nd-10th generation queens obtained from Bombus hypocrita sapporoensis and $B$. terrestris colonies reared year-round in a con- trolled- climate room $\left(28^{\circ} \mathrm{C}, 65 \%\right.$ relative humidity, and continuous darkness) at the Department of Agricultural Biology, National Academy of Agricultural Science, Republic of Korea.

\section{Indoor rearing}

We followed a basic colony-rearing technique described previously (Yoon et al., 2002). The queens were reared in three types of cardboard (1.5-mm thick) boxes for nest initiation $(10.5 \times 14.5 \times 6.5 \mathrm{~cm}$ : small box $)$, colony foundation $(21.0 \times 21.0 \times 15.0 \mathrm{~cm}$ : medium box $)$, and colony maturation $(24.0 \times 27.0 \times 18.0 \mathrm{~cm}$ : large box $)$. Each box had a wire net window on its lid for ventilation. The sizes of these windows were $5.5 \times 6.5 \mathrm{~cm}, 7.0 \times 14.0 \mathrm{~cm}$ and $10.0 \times 20.0 \mathrm{~cm}$, respectively. The queens were first confined individually in small boxes for colony initiation and remained there until oviposition. When the adults emerged from the first brood, the nest was transferred to a medium box for colony foundation and was left there until the number of workers reached 50 . The nest was then moved to the large box for further colony development. Sugar solution and pollen dough were provided ad libitum. The pollen dough was made from sugar solution and fresh pollen collected from an apiary $(\mathrm{v}: \mathrm{v}=1: 1)$.

Hybridization and colony development of Bombus spp. To verify the interspecific hybridization of Bombus spp., experimental regimes of $B$. $h$. sapporoensis queen $\times B$. terrestris male or $B$. terrestris queen $\times B$. h. sapporoensis male were used. The bumblebees used for these experiments were taken from reared colonies. Newly emerged virgin queens were collected directly from the nest and maintained separately in cages until the copulation experiments took place. This procedure isolated the queen from the males' odor and from any potential nest copulations. The unmated males were taken from the nest before the experiment and introduced into the cage. Like the queen, they were isolated from the odor of their future partner (the queen). For the mating experiments, thirty 6- to 7-day-old virgin queens and ninety 10- to 11-day-old males were introduced into a wooden mating arena $(55 \times 65 \times 40 \mathrm{~cm})$ covered with wire mesh. The mating experiment started at 09:00 and ended at 17:00. The environmental conditions used in the mating room were as follows: intensity of illumination, over 2,000 Lux; UV, $11-13 \mu \mathrm{W} / \mathrm{cm}^{2}$; temperature, $23-24^{\circ} \mathrm{C}$; and humidity, $60-65 \%$. If copulation occurred, the mated queen and the male were moved to a small cardboard box and were monitored during the duration of mating. To investigate competition for copulations when $B$. $h$. sapporoensis queens and $B$. terrestris queens were placed with $B$. $h$. sapporoensis males and $B$. terrestris males, $10 \mathrm{~B}$. h. sapporoensis queens and $10 \mathrm{~B}$. ter- 
Table 1. Mating percentage and mating duration of Bombus spp

\begin{tabular}{|c|c|c|c|c|c|c|c|c|}
\hline $\begin{array}{l}\text { Surveyed } \\
\text { items }\end{array}$ & $\mathrm{n}$ & $\begin{array}{c}\text { B.h. sapporoensis } \\
\text { queen } \times \\
\text { B.h. sapporoensis male }\end{array}$ & $\mathrm{n}$ & $\begin{array}{l}\text { B.h. sapporoensis } \\
\text { queen } \times \\
\text { B. terrestris male }\end{array}$ & $\mathrm{n}$ & $\begin{array}{l}\text { B. terrestris queen } \times \\
\text { B. terrestris male }\end{array}$ & $\mathrm{n}$ & $\begin{array}{l}\text { B. terrestris queen } \times \\
\text { B.h. sapporoensis } \\
\text { male }\end{array}$ \\
\hline Mating (\%) & 30 & 36.7 & 30 & 13.3 & 30 & 86.7 & 30 & 43.3 \\
\hline $\begin{array}{l}\text { Mating duration } \\
\text { (min) }\end{array}$ & 52 & $29.5 \pm 9.6 \mathrm{abc}$ & 6 & $35.2 \pm 7.7 \mathrm{ab}$ & 45 & $34.5 \pm 10.8 \mathrm{a}$ & 27 & $24.8 \pm 6.0 \mathrm{bc}$ \\
\hline
\end{tabular}

Statistical analysis: mating percentage, Chi-square test: $\chi^{2}=34.074, \mathrm{df}=3, P=0.0001$; mating duration, Tukey's pairwise comparison test, $\mathrm{F}=6.68$, df $=3,126, P=0.0001$.

restris queens were simultaneously put in one cage with 40 B. h. sapporoensis males and 40 B. terrestris males. Competition for copulations between $B$. $h$. sapporoensis males and $B$. terrestris males was also investigated. Sixty males of each species were placed simultaneously in one cage with $60 \mathrm{~B}$. h. sapporoensis queens and $60 \mathrm{~B}$. terrestris queens. In both experiments, the queens were narcotized two days after mating with $99 \% \mathrm{CO}_{2}$ for $30 \mathrm{~min}$ for two consecutive days in a flask to stimulate diapause (Yoon et al., 2003) and reared to investigate egg-laying characteristics, colony development, and emergence of the next generation. Mating duration was recorded as the time ( $\min$ ) when the queen first engaged in a copula with a male until the time the copula ended. The actual copulation can be easily observed because the male, while freely hanging behind the queen, grasps and firmly holds the female outer genitalia. The developmental ability of each colony was estimated by the percentage of oviposition, colony foundation, and progeny-queen foundation. Colony foundation was indicated by the emergence of more than 50 workers from a colony. The preoviposition period was measured as the number of days until the first oviposition. Queens that did not oviposit within 60 days were excluded from the counts of the number of oviposited colonies.

\section{Statistical analysis}

Chi-square tests and one-way ANOVA (MINITAB Release 13 for Windows, 2000) were used for statistical analysis. The Chi-square test was used to compare the percentage of mating, oviposition, and death within one month for the hybridization of Bombus spp. Tukey's pairwise comparison test (one-way ANOVA) was used to examine the mating durations and preoviposition period for the hybridization of Bombus spp.

\section{Results and Discussion}

Hybridization of B. h. sapporoensis and B. terrestris

We investigated possible hybridization between the
Korean native bumblebee $B$. $h$. sapporoensis and the imported European bumblebee B. terrestris (Table 1). The mating percentage for $B$. terrestris $(86.7 \%)$ was greater than the mating percentage of $B$. terrestris queens placed with $B$. h. sapporoensis males (43.3\%). The interspecific mating percentage was lower than the intraspecific mating percentage. A similar effect was seen in the interspecific mating of $B$. $h$. sapporoensis queens with $B$. terrestris males (13.3\%). The intraspecific mating percentage of $B$. terrestris was 2.4-fold higher than that of $B$. h. sapporoensis $(36.7 \%)$. Interestingly, the mating percentage for $B$. terrestris queens with $B$. h. sapporoensis males was $6.6 \%$ higher than that for the intraspecific mating of $B$. ignitus. There was a statistically significant difference in the mating percentages of intraspecific and interspecific crosses of B. h. sapporoensis and B. terrestris (Chi-square test: $\chi^{2}$ $=34.074$, d.f. $=3, P=0.0001$ ).

As we have shown in the competitive copulation experiment, the mating of $B$. h. sapporoensis males with $B$. terrestris queens is much more frequent (1.2-fold) than with $B$. h. sapporoensis queens. These observations indicate that $B$. h. sapporoensis males mate with $B$. terrestris queens and suggest that the mating behavior of $B$. terrestris queens may potentially interfere with intraspecific $B$. h. sapporoensis matings. Similar results were reported in the interspecific hybridization of B. ignitus and B. terrestris (Ono, 1997; Yoon et al., 2009). Furthermore, our results indicate that $B$. terrestris queens have more successful matings with $B$. h. sapporoensis males than $B$. $h$. sapporoensis queens have with their own males. Although this effect may result from the rearing of $B$. terrestris in the laboratory compared with $B$. h. sapporoensis, our finding suggests that $B$. terrestris queens will have a competitive impact on the mating of $B$. $h$. sapporoensis queens. Consequently, competition could have a negative effect on the intraspecific matings of $B$. h. sapporoensis. In addition, the intraspecific mating rate of $B$. terrestris was 2.4-fold higher than that of $B$. h. sapporoensis. In view of the mating ability of $B$. terrestris, it is probable that $B$. terrestris will compete significantly with $B$. h. sapporoensis and could be superior to $B$. $h$. sapporoensis in 
competition for nest sites and food resources (Hingston and McQuillan, 1999; Hingston et al., 2002; Matsumura et al., 2004; Velthuis and van Doorn, 2006).

The mating duration in intraspecific and interspecific crosses of $B$. h. sapporoensis and B. terrestris was also investigated. The mating duration in intraspecific and interspecific crosses of $B$. h. sapporoensis and B. terrestris is about $25-35 \mathrm{~min}$ (Table 1). The mating duration in the intraspecific mating of $B$. $h$. sapporoensis tended to be 5 min shorter than that of the intraspecific mating of $B$. terrestris. The hybridization of a $B$. h. sapporoensis queen and $B$. terrestris with a $B$. terrestris male has a longer mean mating duration (34.5-35.2 min) than that of a $B . h$. sapporoensis queen and $B$. terrestris with a $B$. h. sapporoensis male (24.8-29.5 min). There was a statistical difference in the mating duration of the Bombus spp. (oneway ANOVA, $\mathrm{F}=6.68$, d.f. $=3,126, P=0.0001$ ). This result indicates that in the hybridization of $B$. $h$. sapporoensis and $B$. terrestris, the mating duration shows a tendency toward the duration associated with $B$. terrestris. A similar result was seen in the interspecific hybridization of B. ignitus and B. terrestris (Yoon et al., 2009). The mating duration has been recorded for a number of bumblebee species (Duvoisin et al., 1999; Brown et al., 2002).

We sought to determine whether the frequency of interspecific hybridization of $B$. terrestris queens with $B . h$. sapporoensis males and $B$. h. sapporoensis queens with $B$. terrestris males is higher than the frequency of intraspecific mating of $B$. $h$. sapporoensis and B. terrestris queens. To investigate this question, $B$. $h$. sapporoensis queens and $B$. terrestris queens were placed simultaneously in one cage with $B$. h. sapporoensis males and $B$. terrestris males. We found that the mating percentage for the interspecific mating of $B$. terrestris queens with $B$. h. sapporoensis males was $40.0 \%, 1.3$-fold higher than that of the intraspecific mating of $B$. h. sapporoensis queens, though there was no statistically significant difference (Chi-square test: $\chi^{2}=0.220$, d.f. $=1, P=0.639$ ) (Fig. 1A). However, the mating percentage for the interspecific hybridization of $B$. h. sapporoensis queens with $B$. terrestris males was $40.0 \%, 2.3$-fold lower than that of the intraspecific mating of $B$. terrestris queens (Chi-square test: $\chi^{2}=5.495$, d.f. $=1, P=0.019$ ) (Fig. 1B). The mating percentage for the interspecific mating of $B$. h. sapporoensis queens with $B$. terrestris males was $26.7 \%, 2.7$-fold higher than that of the intraspecific matings of $B$. h. sapporoensis queens (Chi-square test: $\chi^{2}=2.783$, d.f. $=1, P$ $=0.095$ ) (Fig. 2A). Like the mating percentage for the interspecific mating of $B$. $h$. sapporoensis queens with $B$. terrestris, the mating percentage for the interspecific mating of $B$. terrestris queens with $B$. h. sapporoensis males was $16.7 \%, 5.0$-fold lower than that of the intraspecific
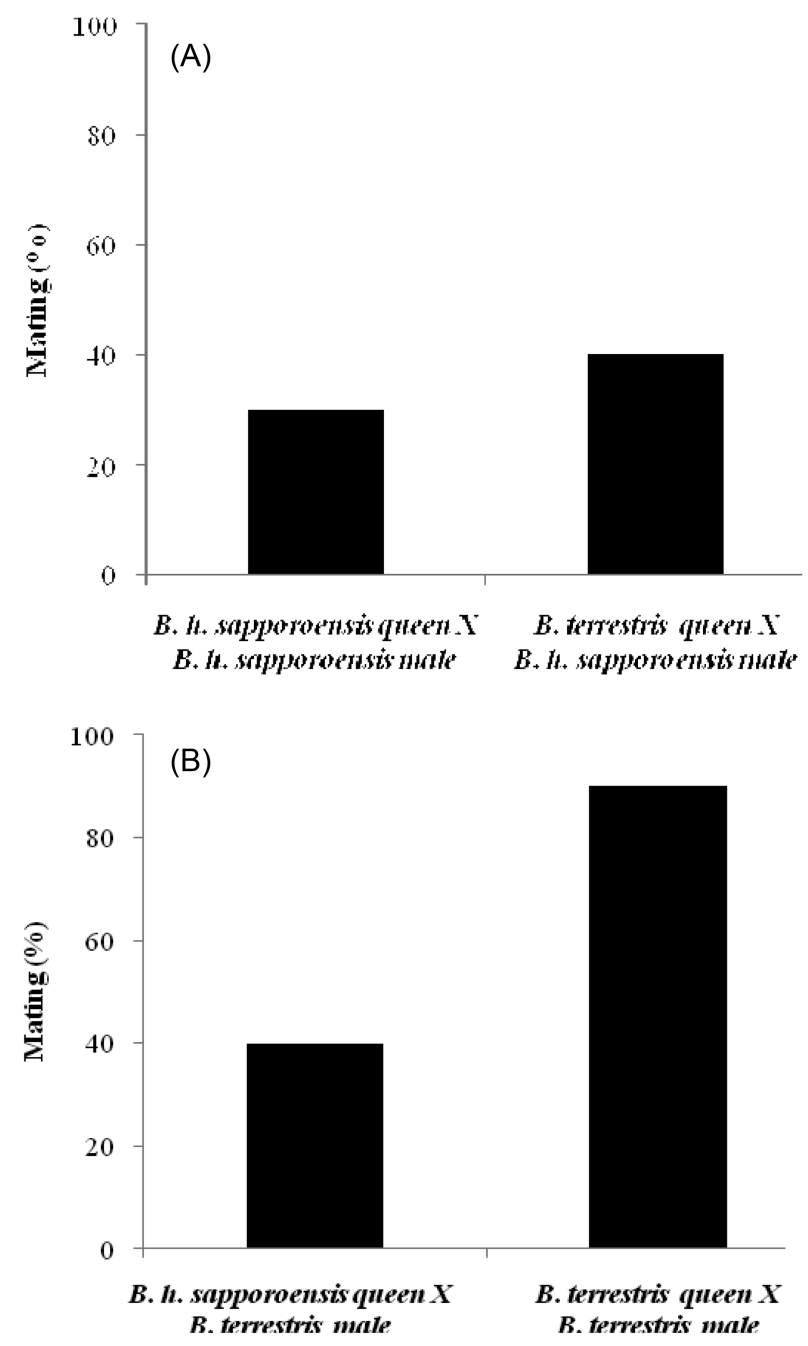

Fig. 1. Competitive copulation of $B$. $h$. sapporoensis queens and $B$. terrestris queens with $B$. h. sapporoensis males (A) and $B$. terrestris males (B). Ten queens of each species were simultaneously put in one cage with 40 males. Statistical analysis: mating percentage of $B . h$. sapporoensis queens and $B$. terrestris queens with $B$. terrestris males, Chi-square test $\chi^{2}=5.495$, d.f. $=1, P=0.019$.

matings of $B$. terrestris queens (Chi-square test: $\chi^{2}=$ 29.433, d.f. $=1, P=0.0001$ ) (Fig. 2B). The above results indicate that the $B$. terrestris queen and male are superior to the $B$. h. sapporoensis queen and male in mating. Kanbe et al. (2008) reported that nearly $30 \%$ of the queens of the Japanese native species $B$. h. sapporoensis and $B . h$. hypocrita were estimated to copulate with $B$. terrestris males in the field and suggested that indigenous bumblebees could suffer from genetic deterioration through the production of hybrids with the introduced species.

Queens of mated Bombus spp., after treatment with carbon dioxide to stimulate diapause (Yoon et al., 2003), 

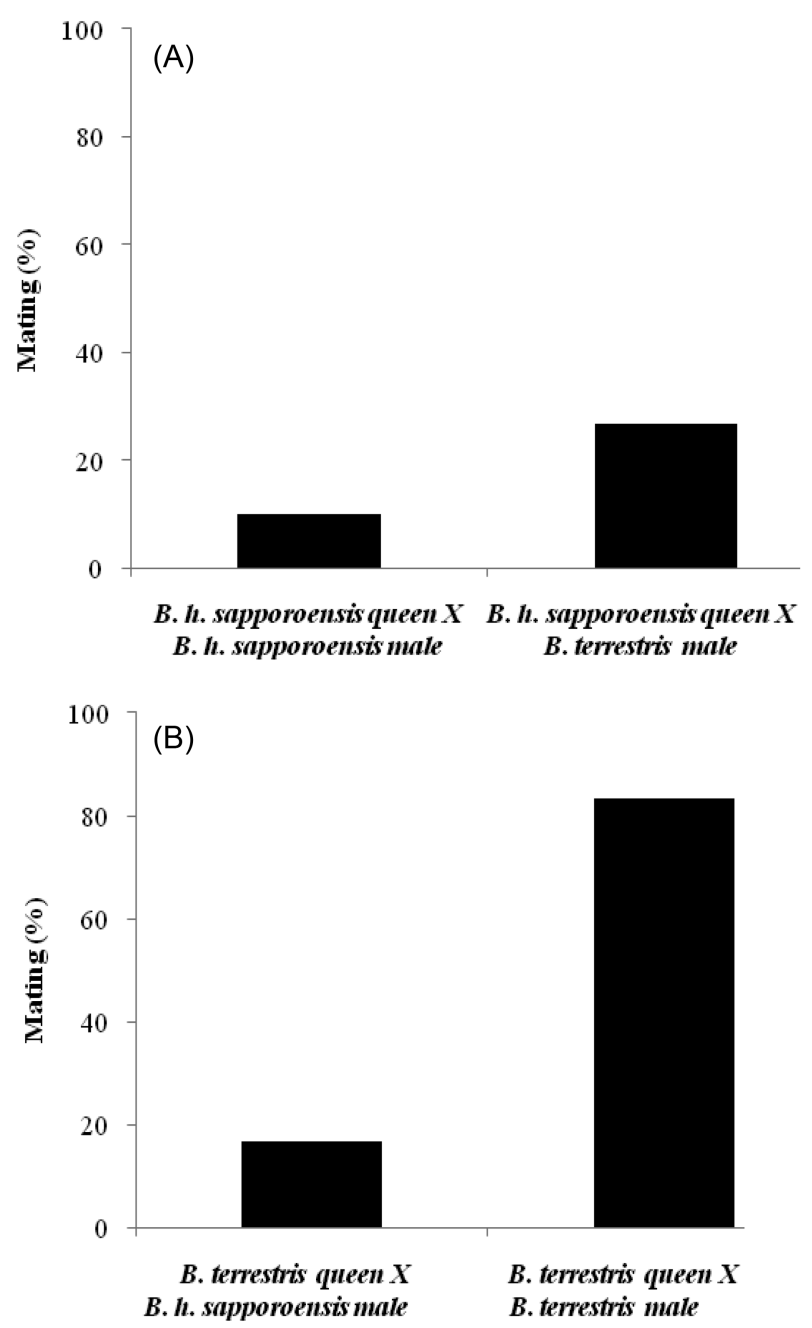

Fig. 2. Competitive copulation of $B$. $h$. sapporoensis males and $B$. terrestris males with $B$. h. sapporoensis queens (A) and $B$. terrestris queens (B). Sixty males of each species were simultaneously put in one cage with 60 queens. Statistical analysis: mating percentage of the B.h. sapporoensis male and B. terrestris male with B.terrestris queen, Chi-square test $\chi^{2}=29.433$, d.f. $=1, P=0.001$.

were reared to determine the presence of oviposition. We surveyed oviposition percentages and the preoviposition periods of intraspecifically and interspecifically mated Bombus spp. (Fig. 3). The oviposition percentage was highest in the $B$. terrestris queen $\times B$. terrestris male cross $(90.0 \%)$, followed by the $B$. terrestris queen $\times B$. h. sapporoensis $(80.0 \%)$ cross, the $B$. $h$. sapporoensis queen $\times$ B. h. sapporoensis male $(66.7 \%)$ cross, and the $B$. $h$. sapporoensis queen $\times B$. terrestris male cross $(20.0 \%)$. It is noteworthy that the oviposition percentage of $B$. terrestris queens mated with $B$. h. sapporoensis males was 1.2-fold higher than that of intraspecifically mated $B$. h. sapporoensis queens and was 4-fold higher than that of $B . h$.

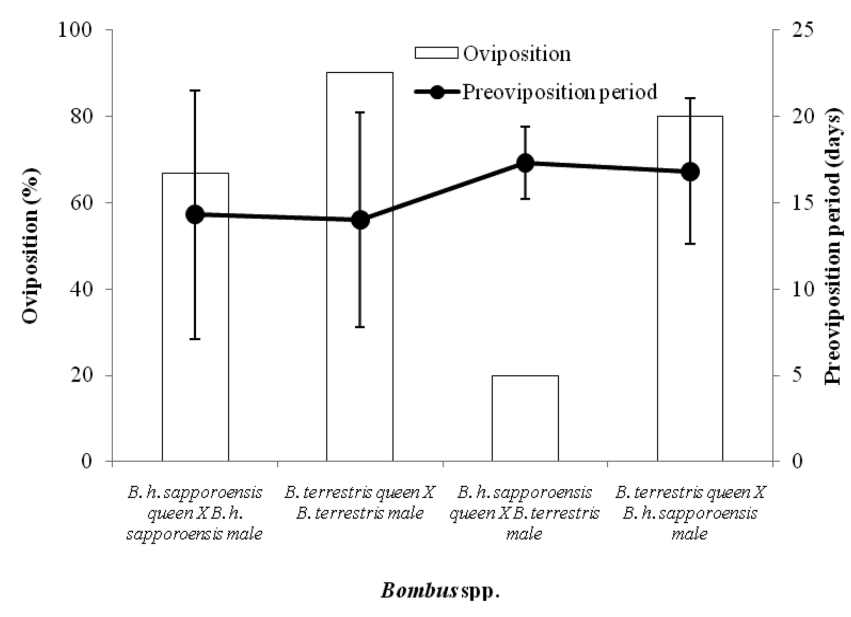

Fig. 3. Oviposition percentage and preoviposition period of hybridized queens. Queens were reared after $\mathrm{CO}_{2}$ treatment to simulate diapause at 2 days after mating. For the statistical analysis, a Chi-square test was used: $\chi^{2}=37.511$, d.f. $=3, P=$ 0.0001 for the percentage of oviposition.

sapporoensis queens mated to $B$. terrestris males. There was a statistically significant difference in the oviposition percentages of the two Bombus spp. (Chi-square: $\chi^{2}=$ 37.511 , d.f. $=3, P=0.0001)$. However, there was no significant difference in the preoviposition period (14-17 days) for the Bombus spp.

\section{Colony development in hybridized Bombus spp.}

We investigated the percentage of death within 1 month post-rearing, the percentage of adult emergence, and the percentage of colony foundation for colony development (Table 2). The percentage of death within 1 month after rearing was $6.6 \%$ and $9.1 \%$ for intraspecifically mated $B$. terrestris queens and interspecifically mated $B$. terrestris queens, respectively. Compared with the progeny from intraspecific matings of B. h. sapporoensis, $13.3 \%$ of which died within the month, $70.0 \%$ of the progeny from $B$. h. sapporoensis queens mated with $B$. terrestris males died within 1 month after rearing. The percentage of death in intraspecific matings of $B$. $h$. sapporoensis was 2.0 -fold higher than that in intraspecific matings of $B$. terrestris (Chi-square: $\chi^{2}=0.741$, d.f. $=1, P=0.389$ ). Furthermore, the percentage of death of the progeny of $B . h$. sapporoensis queens mated with $B$. terrestris males was 5.3-fold higher than that of $B$. terrestris queens mated with $B . h$. sapporoensis males (Chi-square: $\chi^{2}=24.721$, d.f. $=1, P=$ $0.0001)$. The percentage of death within 1 month after rearing was significantly affected by interspecific hybridization in the maternal line of $B . h$. sapporoensis (Chisquare: $\chi^{2}=45.138$, d.f. $\left.=3, P=0.0001\right)$. A similar result was seen in the interspecific mating of $B$. ignitus and $B$. 
Table 2. Colony development of hybridized Bombus spp

\begin{tabular}{|c|c|c|c|c|c|c|}
\hline Bombus spp. & $\mathrm{n}$ & $\begin{array}{l}\text { Death within } \\
1 \text { month after } \\
\text { rearing } \\
(\%)\end{array}$ & $\begin{array}{c}\text { Worker } \\
\text { emergence } \\
(\%)\end{array}$ & $\begin{array}{c}\text { Male } \\
\text { emergence } \\
(\%)\end{array}$ & $\begin{array}{l}\text { Progeny- } \\
\text { queen } \\
\text { production } \\
(\%)\end{array}$ & $\begin{array}{c}\text { Colony } \\
\text { foundation } \\
(\%)\end{array}$ \\
\hline $\begin{array}{l}\text { B.h. sapporoensis queen } \times \\
\text { B.h. sapporoensis male }\end{array}$ & 30 & 13.3 & 43.3 & 56.7 & 10.0 & 13.3 \\
\hline $\begin{array}{l}\text { B. terrestris queen } \times \\
\text { B. terrestris male }\end{array}$ & 30 & 6.6 & 76.7 & 83.3 & 33.3 & 43.3 \\
\hline $\begin{array}{l}\text { B.h. sapporoensis queen } \times \\
\text { B. terrestris male }\end{array}$ & 30 & 70.0 & 0.0 & 16.7 & 0.0 & 0.0 \\
\hline $\begin{array}{c}\text { B. terrestris queen } \times \\
\text { B.h. sapporoensis male }\end{array}$ & 33 & 9.1 & 3.0 & 36.4 & 0.0 & 0.0 \\
\hline
\end{tabular}

Statistical analysis: Chi-square test $\chi^{2}=45.128, \mathrm{df}=3, P=0.0001$ for the percentage of death within 1 month.

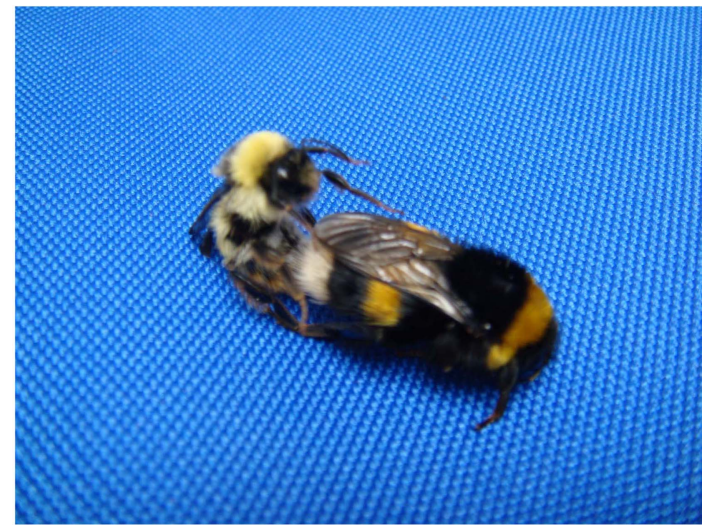

(A)

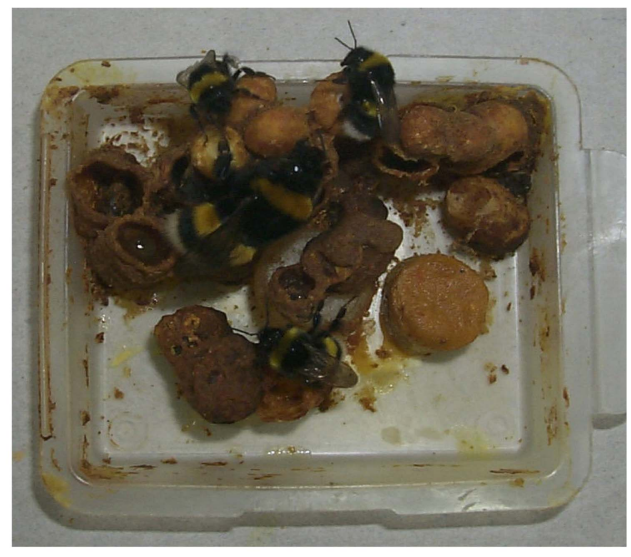

(B)

Fig. 4. Copulation of the $B$. terrestris queen with a $B$. $h$. sapporoensis male (A) and colony produced from a $B$. terrestris queen hybridized with a $B$. h. sapporoensis male (B).

terrestris (Yoon et al., 2009).

The results for adult emergence showed that $B$. terrestris queens hybridized with $B$. h. sapporoensis males produced workers resembling $B$. terrestris workers and males resembling $B$. terrestris males, although the percentage of worker emergence was low (3.0\%) (Table 2, Fig. 4A and B). In contrast, B. h. sapporoensis queens hybridized with B. terrestris males only produced males (16.7\%) (Table $2)$. The phenotypes of the hybrids were easily differentiated by morphological characteristics and their similarities with $B$. terrestris or $B$. h. sapporoensis identified (data not shown). These morphological characteristics showed that the hybrid phenotype was dependent on the maternal line. Our results show that interspecifically hybridized queens of $B$. ignitus or B. terrestris produced hybrids but did not form a colony. The mating, survival and oviposition percentages of $B$. terrestris queens hybridized with $B$. ignitus males were all higher than the corresponding values for the intraspecific mating of $B$. ignitus.

The interspecific hybridization of Bombus spp. has already been reported. A previous study has shown that $B$. terrestris mated with $B$. ignitus, $B$. hypocrita hypocrita and $B$. h. sapporoensis and that no hybrid females were obtained (Ono, 1997). In contrast, Mitsuhata and Ono (1996) reported that a $B$. h. sapporoensis queen hybridized with a $B$. terrestris male produced hybrid workers and new queens and that the hybrid queen copulated with a male $B$. terrestris but did not initiate a nest. A recent study has shown that the interspecific mating of $B$. terrestris and $B$. $h$. sapporoensis produces inviable hybrids (Kanbe et al., 2008). The results of these previous studies are consistent with our observation that hybridized queens of $B . h$. sapporoensis or $B$. terrestris produced hybrids and that if a hybrid male $B$. terrestris copulated with a virgin queen of $B$. terrestris, the queen oviposited but did not initiate a nest. We did not observe ovipoisition by hybrid workers, but a previous study found that the hybrid workers oviposited unfertilized eggs, which developed into adult second-generation males (Mitsuhata and Ono, 1996). 
We have shown that hybridization can occur between $B$. $h$. sapporoensis and $B$. terrestris. Our study identified the competitive effect of $B$. terrestris on intraspecific matings of naturally occurring $B$. h. sapporoensis by demonstrating that $B . h$. sapporoensis males mate preferentially with $B$. terrestris queens. In addition, we hypothesized that such interspecies hybridization could cause genetic contamination of the native bumblebees. Further studies of interspecific hybridization and the development of any progeny produced will ultimately provide important information about the ecological impacts of introduced Bombus spp. on native bumblebees.

\section{Acknowledgement}

This study was carried out with the support of "Research Program for Agricultural Science \& Technology Development (PJ006801032011)," National Academy of Agricultural Science, Rural Development Administration, Republic of Korea.

\section{References}

Brown MJF, Baer B, Schmid-Hempel R, Schmid-Hempel P (2002) Dynamics of multiple-mating in the bumble bee Bombus hypnorum. Insectes Sociaux 49, 315-319.

Chittka L, Ings TC, Raine NE (2004) Chance and adaptation in the evolution of island bumblebee behavior. Popul Ecol 46, 243-251.

Dafni A (1998) The threat of Bombus terrestris spread. Bee World 79, 113-114.

Dag A, Kammer Y (2001) Comparison between the effectiveness of honeybee (Apis millifera) and bumblebee (Bombus terrestris) as pollinators of greenhouse sweet pepper (Capsicum annuum). Am Bee J 141, 447-448.

Dafni A, Shimida A (1996) The possible ecological implications of the invasion of Bombus terrestris (L.) (Apidae) at Mt Carmel, Israel; in The conservation of bees. Matheson A.C. (ed.), pp. 183-200, The Linnean Society of London and The International Bee Research Association, London, UK.

Donovan BJ, Wier SS (1978) Development of hives for field population increase, and studies on the life cycles of the four species of introduced bumble bees in New Zealand. NZ J Agric Res 21, 733-756.

Duvoisin N, Baer B, Schmid-Hempel P (1999) Sperm transfer and male competition in the bumblebee, Bombus terrestris L. Anim Behav 58, 743-749.

Estoup A, Solignac M, Cornuet JM, Goudet J, Scholl A (1996) Genetic differentiation of continental and island populations of Bombus terrestris (Hymenoptera: Apidae) in Europe. Moi Ecol 5, 19-31.
Free JB (1993) Insect pollination of crops. 2nd ed., Academic Press, London.

Goka K (1998) Influences of invasive species on native species: Will the European bumblebee, Bombus terrestris bring genetic pollution into the Japanese native species?. Bull Biogeogr Soc Jap 53, 91-101.

Greenleaf S, Kremen C (2006) Wild bee species increase tomato production but respond differently to surrounding land use in Northern California. Biol Conserv 133, 81-87.

Hingston AB, Marsden-Smedley J, Driscoll DA, Corvett S, Fenton J, Aanderson R, Plowman C, Mowling F, Jenkin M, Matsui K, Bonham KJ, Iowski M, McQullian PB, Yaxley B, Reid T, Storey D, Poole L, Mallick SA, Fitzgerald N, Kirkpatrick JB, Febey J, Harwood AG, Michaels JF, Russell MJ, Black PG, Emmerson L, Visoiu M, Morgan J, Breen S, Gates SS, Bantich MN, Desmarchelier JM (2002) Extent of invasion of Tasmanian native vegetation by the exotic bumblebee Bombus terrestris (Apidea: Apidae). Aust J Ecol 27, 162-172.

Hingston AB, McQullian PB (1998) Does the recently introduced bumblebee Bombus terrestris (Apidae) threaten Australian ecosystems?. Aust J Ecol 23, 539-549.

Hingston AB, McQullian PB (1999) Displacement of Tasmanian native megachilid bees by the recently introduced bumblebee Bombus terrestris (Linnaeus, 1758) (Hymenoptera: Apidae). Aust J Zool 47, 59-65.

Inoue $\mathrm{MN}$, Yokoyama J (2010) Competition for flower resources and nest sites between Bombus terrestris (L.) and Japanese native bumblebees. Appl Entomol Zool 45, 29-35.

Inoue MN, Yokoyama J, Washitani I (2008) Displacement of Japanese native bumblebees by the recently introduced Bombus terrestris (L.) (Hymenopter: Apidae). J Insect Conserv 12, 135-146.

Iwasaki M (1995) Introduction of commercial bumblebees into Japan. Honeybee Sci 16, 17-21.

Kanbe Y, Okada I, Yoneda M, Goka K, Tsuchida K (2008) Interspecific mating of the introduced bumblebee Bombus terrestris and the native Japanese bumblebee Bombus hypocrite sapporoensis results in inviable hybrids. Naturwissenschaften 95, 1003-1008.

Matsumura C, Yokoyama J, Washitani I (2004) Invasion status and potential ecological impact of an invasive alien bumblebee, Bombus terrestris L. (Hymenoptera: Apidae) naturalized in Southern Hokkaido, Japan. Global Environ Res 8, 51-66.

Minitab incorporated company (2000) Minitab user's guide, Minitab Inc. USA

Mitsuhata M (2000) Pollination of crops with bumblebee colonies in Japan. Honeybee Sci 21, 17-25.

Mitsuhata M, Ono M (1996) Hybridization between Japanese and European bumblebees (Bombus spp.). Proceedings of the 7th International Pollination Symposium held in Lethbridge, Alberta, Canada.

Morandin LA, Winston ML (2005) Wild bee abundance and seed production in conventional, organic, and genetically 
modified canola. Ecol Appl 15, 871-881.

Ono M (1997) Ecological implications of introduced Bombus terrestris, and significance of domestication of Japanese native bumblebees (Bombus spp.). Proceedings of International Workshop on Biological Invasions of Ecosystem by Pests and Beneficial Organisms held in National Institute of Agro-Environmental Sciences, Ministry of Agriculture, Forestry and Fisheries, pp. 242-252, Tsukuba, Japan.

Ono M, Wada T (1996) The world of the bumblebee. Japanese association for plant protection, p. 132, Tokyo, Japan.

Rhymer JM, Simberloff D (1996) Extention by hybridization and introgression. Ann Rev Ecolog Syst 27, 83-109.

Ruz L, Herrera R (2001) Preliminary observations on foraging activities of Bombus dahlbomii and Bombus terrestris (Hym: Apidae) on native and non-native vegetation in Chile. Acta Hortic 561, 165-169.

Semmens TD, Turner E, Buttermore R (1993) Bombus terrestris (L.) (Hymenoptera: Apidae) now established in Tasmania. J Aust Entomol Sci 32, 346.

Velthuis HHW, van Doorn A (2006) A century of advances in bumblebee domestication and the economic and environmental aspects of its commercialization for pollination. Apidologie 37, 421-451.

Washitani I (1998) Conservation-ecological issues of the recent invasion of Bombus terrestris into Japan. Jap J Ecol $48,73-78$.
Winfree R, Griswold T, Kremen C (2007) Effect of human disturbance on bee communities in a forested ecosystem. Conserv Biol 21, 213-223.

Yoneda M, Tsuchida K, Goka K (2008) Ecological risks of commercialized bumblebees and the Invasive Alien Species Act. Jap J Appl Entomol Zool 52, 47-62.

Yoon HJ, Kim SE, Kim YS (2002) Temperature and humidity favorable for colony development of the indoor-reared bumblebee, Bombus ignitus. Appl Entomol Zool 37, 419-423.

Yoon HJ, Kim SE, Kim YS, Lee SB (2004) Colony developmental characteristics of the bumblebee queen, Bombus ignitus by the first oviposition day. Int J Indust Entomol 8, 139143.

Yoon HJ, Kim SY, Lee KY, Lee SB, Park IG, Kim I (2009) Interspecific hybridization of the bumblebees Bombus ignitus and B. terrestris. Int J Indust Entomol 18, 41-48.

Yoon HJ, Kim SE, Lee SB, Park IG (2003) Effect of $\mathrm{CO}_{2}$-treatment on oviposition and colony development of the bumblebee, Bombus ignitus. Korean J Appl Entomol 42, 139-144.

Yoon HJ, Lee KY, Park IG, Kim MA (2010) Temperature and humidity favorable for colony development of Korean Native Bumblebee Bombus hypocrita sapporoensis. Korean J Apiculture 25, 83-89.

Yoon HJ, Mah YI, Lee MY, Park IG, Bilinski M (1999) Ecological characteristics of Bombus ignitus Smith in Korea. Korean J Appl Entomol 38, 101-107. 